\title{
ZNF703 promotes tumor cell proliferation and invasion and predicts poor prognosis in patients with colorectal cancer
}

\author{
FENG MA $^{1 *}$, LIHONG BI ${ }^{2 *}$, GONGLI YANG $^{2,3^{*}}$, MENGNAN ZHANG $^{4}$, CUIPING LIU $^{5}$, \\ YINGYING ZHAO ${ }^{2}$, YADONG WANG $^{2}$, JIDE WANG $^{2}$, YANG BAI $^{2}$ and YALI ZHANG ${ }^{2}$
}

\begin{abstract}
${ }^{1}$ Department of Gastroenterology, The First Affiliated Hospital of Jinan University, Guangzhou, Guangdong 510630; ${ }^{2}$ Guangdong Provincial Key Laboratory of Gastroenterology, Department of Gastroenterology, Nanfang Hospital, Southern Medical University, Guangzhou, Guangdong 510515; ${ }^{3}$ Department of Gastroenterology, Taihe Hospital, Hubei University of Medicine, Shiyan, Hubei 442000; ${ }^{4}$ Department of Gastroenterology, The First Affiliated Hospital of Guangzhou Medical University, Guangzhou, Guangdong 510210; ${ }^{5}$ Department of Emergency Medicine, Zhujiang Hospital, Southern Medical University, Guangzhou, Guangdong 510515, P.R. China
\end{abstract}

Received March 14, 2014; Accepted May 5, 2014

DOI: $10.3892 /$ or.2014.3313

\begin{abstract}
Zinc finger protein 703 (ZNF703), identified as an oncogene in luminal B breast cancer, is a member of the NET/NlZ family of zinc finger transcription factors. However, the role of ZNF703 in colorectal cancer (CRC) is unknown. We investigated the expression of ZNF703 in paired tumor and corresponding normal tissues using reverse transcriptase-polymerase chain reaction (RT-PCR) and western blot analysis. Immunohistochemistry (IHC) was applied on paraffin-embedded specimens, including 138 CRC tissues, 58 matched normal tissues and 30 paired metastatic lymph node samples. Levels of mRNA $(72.72 \%, 16 / 22)$ and ZNF703 protein expression $(65.38 \%, 17 / 26$, respectively) were upregulated in CRC tissues. IHC staining revealed higher expression of ZNF703 in the CRC tissues $(68 / 138,49.3 \%)$ compared with that in the adjacent normal mucosal tissues $(4 / 58,6.9 \%)(\mathrm{P}<0.001)$. Moreover, high ZNF703 expression was significantly correlated with tumor size, pathological grading, serosal invasion, lymph node metastasis and AJCC stage. CRC patients with relatively low ZNF703 expression had higher survival rates than those with high ZNF703 expression. In addition, we investigated ZNF703 expression in eight CRC cell lines (LS174T, SW480,
\end{abstract}

Correspondence to: Dr Yang Bai or Dr Yali Zhang, Department of Digestive Medicine, Nanfang Hospital, The Southern Medical University, Guangzhou, Guangdong 510515, P.R. China

E-mail: baiyang1030@hotmail.com

E-mail: zyl641531@163.com

${ }^{*}$ Contributed equally

Abbreviations: ZNF703, zinc finger protein 703; CRC, colorectal cancer; NLZ1, NocA-like zinc finger protein 1; $\mathrm{ER}^{+}$, estrogen receptor-positive

Key words: ZNF703, colorectal cancer, prognosis, proliferation, migration
HT29, SW620, DLD1, SW1116, LoVo and CaCo-2) in vitro. The highest ZNF703 expression was detected in the LoVo cell line. RNA interference was used to assess the effects of ZNF703 knockdown in LoVo cells. Knockdown of ZNF703 expression inhibited CRC cell proliferation and migration. Collectively, these results reveal that ZNF703 may act as an oncogene in $\mathrm{CRC}$ and could be considered as a potential therapeutic target for metastatic CRC.

\section{Introduction}

Colorectal cancer (CRC) is the third most frequent malignancy, and represents the fourth most common cause of cancerrelated deaths in the world (1). Despite many advances in the management of early and advanced CRC, the 5-year survival rate of CRC patients remains much lower than expected (2). In the past 20 years, the encouraging improvement in patient outcome has been followed by a plethora of markers of prognosis and response to anticancer therapy, although most are not clinically viable. Nevertheless, several protein and genetic markers that predict prognosis and treatment benefit have been validated (3). The development and progression of CRC is a multistep process that occurs due to the accumulation of numerous genetic alterations, including epigenetic modifications, chromosomal abnormalities and mutations in genes that regulate proliferation, differentiation, apoptosis and angiogenesis (4).

Genes that are altered by amplification and result in concomitant overexpression in tumors are considered candidate oncogenes (5). Human ZNF703 (also known as NocA-like zinc finger protein $1, N L Z 1$ ) is a member of the NET gene family, which is located on chromosome 8 (8p11.23). ZNF703 protein contains six evolutionarily conserved domains, three of which have not been previously described and are specific for NET proteins (6). Several studies have revealed an amplification of the 8p11-12 chromosomal region associated with human breast cancers, particularly with the luminal B subtype (7-16). In addition, two recent studies have strongly 
suggested the possible role of $Z N F 703$ as a potential breast oncogene $(15,16)$. For example, $Z N F 703$ expression is correlated with poor clinical prognosis in estrogen receptor-positive $\left(\mathrm{ER}^{+}\right)$breast cancer patients. Overexpression of ZNF703 induces cell proliferation and interferes with transforming growth factor $\beta$ (TGF- $\beta$ ) signaling in breast epithelial cells (15). Furthermore, ZNF703 is overexpressed in luminal B-type breast cancer cell lines, MDA-MB-134 and HCC1500, while its expression is low in the normal mammary epithelial cell line MCF-10A (17). In addition, relatively high expression of ZNF703 was found in luminal breast cancers, and is associated with an intermediate tumor grade (17), and high ZNF703 mRNA expression is correlated with poor survival in patients with $\mathrm{ER}^{+}$luminal B tumors (18). In addition, Znf703 (Zeppo1, zinc finger elbow-related proline domain protein 1) was identified as a human ZNF703 ortholog in mice, which has been shown to regulate proliferation, migration and cell adhesion in mouse mammary epithelial cells (19). Morever, Znf703 was found to regulate transcription by repressing E-cadherin, Wnt and TGF- $\beta$ reporter expression, and to increase lung metastases in a mouse breast cancer model (19).

Based on these findings, clinical correlations and experimental data, it appears that ZNF703 meets the classical definition of an oncogene in luminal B breast tumors. However, to the best of our knowledge, there are no reports on its role in the development and progression of gastrointestinal malignancies. In the present study, we examined the expression of ZNF703 in primary CRC tissues and, using cell line models, aimed to determine its biological role in CRC.

\section{Materials and methods}

Cell lines. CRC cell lines (LS174T, SW480, HT29, SW620, DLD1, SW1116, LoVo and $\mathrm{CaCo} 2$ ) were obtained from the American Type Culture Collection (ATCC; Manassas, VA, USA). All cells were cultured in RPMI-1640 (HyClone, Logan, UT, USA) supplemented with $10 \%$ fetal bovine serum (FBS; HyClone) in a humidified atmosphere of $5 \% \mathrm{CO}_{2}$ at $37^{\circ} \mathrm{C}$. Protein and RNA samples were extracted from subconfluent cells during the exponential phase of growth.

Patients and samples. Fresh frozen CRC tissue samples and their paired normal mucosal samples $(10-\mathrm{cm}$ distance from the tumor), as well as CRC metastatic lymph nodes, were collected at the Department of General Surgery, Nanfang Hospital, Guangzhou, China. In addition, formalin-fixed and paraffinembedded tumor tissues (including 58 paired normal mucosal tissues) from 138 patients with a pathological diagnosis of CRC, who had undergone colonoscopy at the Department of Gastroenterology between 2008 and 2009, were also included in the present study. All patients had undergone elective surgery for CRC at Nanfang Hospital. The comprehensive set of clinicopathological data were obtained from the Tumor Tissue Bank of Nanfang Hospital. Approval for the study was obtained from the Ethics Committee of Guangzhou Southern Medical University in China, and written informed consent was obtained from all patients.

RNA extraction, reverse-transcription and semi-quantitative $R T-P C R$. Total RNA was extracted from the colorectal speci- mens using TRIzol reagent (Invitrogen, Carlsbad, CA, USA). Reverse transcription was performed in a total volume of $25 \mu \mathrm{l}$ with $3 \mu \mathrm{g}$ of total RNA using a RevertAid First Strand cDNA Synthesis kit (Promega, Madison, WI, USA). Next, $2 \mu \mathrm{l}$ of cDNA was used as a template to amplify the ZFN703 fragment using the following primers (F, 5'-GATCAGGGTCCTG AAGATGC-3' and R, 5'-CCGAGTTGAGTTTGGAGGAG-3') and the GoTaq ${ }^{\circledR}$ Green Mix kit (Promega), under the following conditions: $95^{\circ} \mathrm{C}$ for $2 \mathrm{~min} ; 35$ cycles of $95^{\circ} \mathrm{C}$ for $30 \mathrm{sec}, 56^{\circ} \mathrm{C}$ for $30 \mathrm{sec}$, and $73^{\circ} \mathrm{C}$ for $30 \mathrm{sec}$; with final extension of $73^{\circ} \mathrm{C}$ for $5 \mathrm{~min}$. The GAPDH gene was used as an internal control and amplified using the following primers: F, 5'-TATGATGATA TCAAGAGGGTAGT-3' and R, 5'-TGTATCCAAACTCATT GTCATAC-3'.

Western blot analysis. Tissues and cells were lysed in RIPA. Cytosolic and nuclear extract were prepared with a protocal of ProteoExtract subcellular proteome extraction kit (Calbiochem, Darmstadt, Germany). The supernatant was collected, and the protein concentration was quantified using a protein assay reagent (Bio-Rad Laboratories, Hercules, CA, USA).

After boiling, the proteins $(25 \mu \mathrm{g})$ were separated by polyacrylamide gel electrophoresis (PAGE) under denaturing conditions and transferred to a polyvinylidene fluoride membrane (PVDF) (Millipore Corp., Billerica, MA, USA). Membranes were blocked with $5 \%$ skim milk in TBS containing $0.1 \%$ Tween-20 (TBS-T), and then incubated for $1 \mathrm{~h}$ at room temperature with rabbit polyclonal antibody against human ZNF703 (GTX107721; GeneTex, Inc., Irvine, CA, USA) (1:1,000). Mouse monoclonal anti-GAPDH (CW0100A; CWBIO, Beijing, China) or TOPO I (GTX63013; Genetex Inc., Irvine, CA, USA) was used as loading control. Next, membranes were incubated for $1 \mathrm{~h}$ with a 1:3,000 dilution of horseradish peroxidase-conjugated antirabbit immunoglobulin G (sc-45106; Santa Cruz Biotechnology, Santa Cruz, CA, USA) and anti-mouse immunoglobulin G (sc-2962; Santa Cruz Biotechnology). The membranes were developed with a horseradish peroxidase chemiluminescence detection reagent (ECL Plus System; Millipore Corp.) and then exposed to Hyperfilm ECL (Millipore Corp.).

Immunohistochemical analysis. Formalin-fixed and paraffinembedded tissues were cut into 4- $\mu \mathrm{m}$ sections, deparaffinized with xylene and rehydrated through a graded series of ethanol/water. Next, the slides were subjected to heat-induced antigen retrieval in $10 \mathrm{mM}$ sodium citrate buffer $(\mathrm{pH}$ 6.0) in a water bath for $15 \mathrm{~min}$ at $100^{\circ} \mathrm{C}$. All of the specimens were then preincubated with $10 \%$ normal bovine serum and incubated with a primary polyclonal rabbit antibody against human ZNF703 (1:100) (GTX107721; GeneTex) overnight at $4^{\circ} \mathrm{C}$. The slides were next incubated with a biotinylated goat anti-rabbit immunoglobulin $\mathrm{G}$ antibody, and the reaction products were visualized using diaminobenzidine (DAB; Dako, Carpinteria, CA, USA) with methyl green as a counterstain. For negative controls, the primary antibodies were omitted, but otherwise the protocol was the same.

Immunostaining in each tumor was classified into four categories depending on the intensity of staining of cancer cells as negative (0), weak (1) moderate (2) or strong (3). The percentage of stained cells was determined using the following scale: $<5 \%$ (0), $5-25 \%$ (1), $26-50 \%$ (2), $51-75 \%$ (3) and $>75 \%$ (4). 

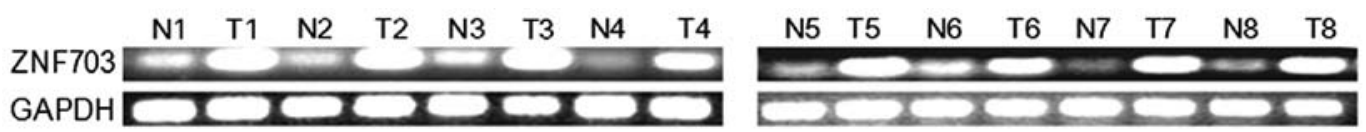

Figure 1. ZNF703 mRNA expression in human CRC tissues and adjacent normal colorectal mucosal tissues as detected by semi-quantitative RT-PCR (GAPDH as internal control). $\mathrm{N}$, normal tissue; $\mathrm{T}$, tumor tissue.

The final score was obtained by multiplying these two values. This resulted in an overall ZNF703 immunohistochemical score of $0,1,2,3,4,6,9$ or 12 . ZNF703 expression was considered low when scores were $\leq 4$, and high when scores were $\geq 6$.

Small interfering RNA (siRNA)-mediated ZNF703 silencing. Expression of human ZNF703 was knocked down with siRNA (Shanghai GenePharma Co., Ltd., Shanghai, China) duplexes 5'-CCACACACUUUGGGCCUAAdTdT-3' (forward) and 5'-dTdTCCACACACUUUGGGCCUAA-3' (reverse) targeting the 3'UTR of endogenous ZNF703. The negative control siRNA 5'-UUCUCCGAACGUGUCACGUTT-3' (forward) and 5'-ACGUGACACGUUCGGAGAATT-3' (reverse) targeting an unknown mRNA sequence was used as a control. Exponential growth phase cells were plated in 6-well plates at a density of $2 \times 10^{5}$ cells $/ \mathrm{ml}$, cultured for $48 \mathrm{~h}$ and transfected with $1 \mu \mathrm{g}$ of siRNA in reduced serum medium (OPTI-MEM-I; Invitrogen) according to the manufacturer's protocol at $30-50 \%$ confluency.

In vitro cell growth assay. Fourty-eight hours after siRNA transfection, cells were prepared at a concentration of $1 \times 10^{4}$ cells/ml. Aliquots $(100 \mu \mathrm{l})$ were dispensed into $96-$ well plates, and cells were incubated for 1, 2, 3, 4, 5 or 6 days. Following incubation, the 3-(4,5-dimethylthiazol-2-yl)-2,5-diphenyltetrazolium bromide (MTT) assay was performed by adding $20 \mathrm{ml}$ of MTT (5 mg/ml; Promega) for $4 \mathrm{~h}$. Next, the supernatants were removed, and $150 \mu \mathrm{l}$ of dimethylsulfoxide (Sigma, St. Louis, MO, USA) was added to each well. Fifteen minutes later, the absorbance value [optical density (OD)] of each well was measured at $490 \mathrm{~nm}$ with a microplate reader. All experiments were repeated three times.

Wound closure assay. Cells transfected with siRNA-ZNF703 and negative control were seeded on 6-well culture plates. When cell confluency reached $\sim 80 \%$ at $48 \mathrm{~h}$ post-transfection, cells were scratched with a sterile $1-\mathrm{ml}$ pipette tip and rinsed with medium to remove any free-floating cells and debris. Complete culture medium was then added, and plates were incubated at $37^{\circ} \mathrm{C}$. Wound closure was observed at 0,24 and $48 \mathrm{~h}$, and representative scrape lines were photographed. Duplicate wells were examined for each condition, and each experiment was repeated three times.

Transwell migration assay. Cells in serum-free medium ( $2 \times 10^{5}$ cells $/ 200 \mu \mathrm{l}$ ) were added to the upper chamber of $8-\mu \mathrm{m}$ pore size Transwell chambers (Corning Star, Cambridge, MA, USA). The bottom chambers contained $10 \%$ FCS as a chemoattractant. Cells were allowed to migrate through the porous membrane for $24 \mathrm{~h}$ at $37^{\circ} \mathrm{C}$. Non-migrating cells that remained on the upper surface of the filter were removed with cotton swabs, and the remaining cells on the lower surface of the filter were fixed with $100 \%$ methanol, stained with crystal violet and counted under a bright field microscope in eight different fields (Nikon E400, x100). Each experiment was independently performed three times.

Statistical analysis. Statistical software SPSS 16.0 (Statistical Package for the Social Sciences; SPSS, Inc., Chicago, IL, USA) was used for statistical analyses. The association between ZNF703 expression and CRC clinicopathological features was analyzed using a $\chi^{2}$ test. The Kaplan-Meier method was used to analyze cumulative survival rate, and differences among groups were estimated using the log-rank test. $\mathrm{P}<0.05$ was considered to indicate a statistically significant result.

\section{Results}

ZNF703 mRNA expression in the CRC tissues. We analyzed ZNF703 mRNA expression in CRC tissues and adjacent normal colorectal mucosal tissues by semi-quantitative RT-PCR. ZNF703 mRNA expression was elevated in the majority of CRC tissues compared with their normal pairs (16/22, 72.72\%); representative RT-PCR results of ZNF703 mRNA expression are presented in Fig. 1.

ZNF703 protein expression in CRC tissues. We also examined expression of ZNF703 protein in the CRC tissues and adjacent normal colorectal mucosal tissues by western blot analysis. Despite the interindividual variations, the expression of ZNF703 protein was significantly upregulated in the CRC tissues (17/26, 65.38\%) (Fig. 2A). The change in ZNF703 protein expression pattern was similar to that observed at the mRNA level.

In order to further investigate ZNF703 protein expression and subcellular localization, we performed IHC analysis in paraffin-embedded CRC tissues, paired adjacent normal colorectal mucosal tissues and metastatic lymph nodes from the same CRC patient. Normal colorectal mucosa typically expressed low ZNF703 protein $(54 / 58,93.10 \%)$. Contrary to this, expression of ZNF703 in CRC tissues was detected in a large proportion of the samples $(68 / 138,49.27 \%)$ and was mainly localized in the nucleus $(58 / 138,42.02 \%)$ and cytoplasm. In addition, the majority of metastatic lymph nodes were positive for ZNF703 (21/23, 91.30\%). Representative results of the ZNF703 IHC analyses are shown in Fig. 2B.

ZNF703 expression and its relation to CRC progression and clinicopathological parameters. To elucidate the role of ZNF703 in the progression of $\mathrm{CRC}$, we examined the correlation between ZNF703 expression and clinicopathological features of the CRC patients including age, gender, tumor size, tumor location, pathological differentiation, serosal invasion, lymph node metastasis and AJCC stage (Table I). Expression of ZNF703 was closely 
A

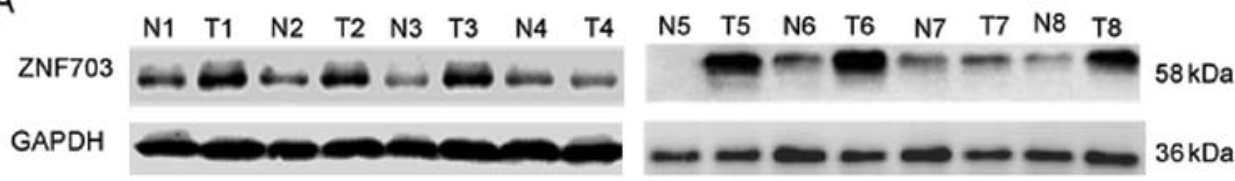

B
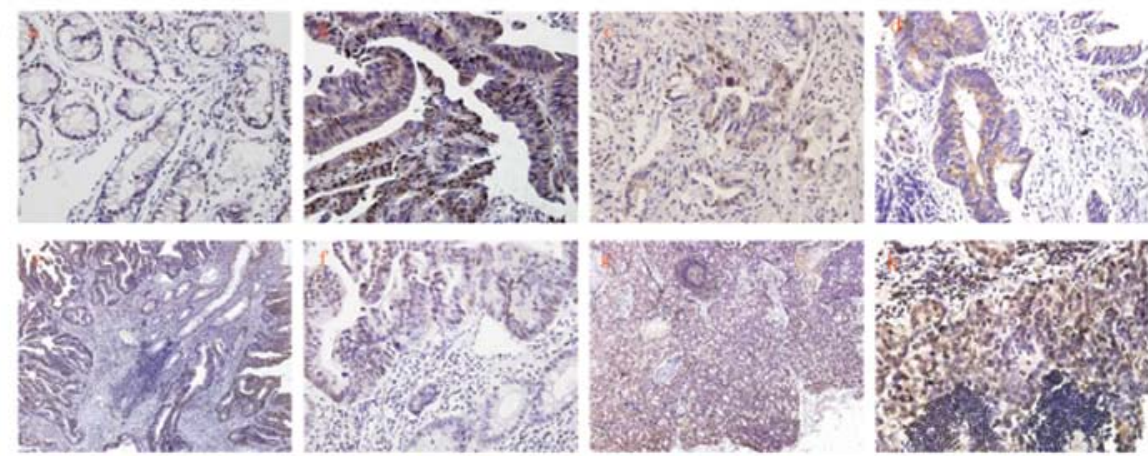

Figure 2. ZNF703 protein expression in human normal colorectal mucosa, CRC tissues and metastatic lymph nodes. (A) Human colorectal carcinoma tissues (T1-T8) and adjacent normal colorectal mucosal tissues (N1-N8) were analyzed by western blot analysis. (B) Immunohistochemical analysis of ZNF703 protein expression: (a) Negative expression in normal colorectal mucosa (original magnification, $\mathrm{x} 400$ ); (b) strong positive nuclear and weak cytoplasmic expression in CRC (original magnification, $\mathrm{x} 400$ ); (c) moderate positive nuclear and weak cytoplasmic expression (original magnification, $\mathrm{x} 400$ ); (d) moderate positive cytoplasmic expression in CRC cells (original magnification, $\mathrm{x} 400$ ); (e and f) moderate-strong positive nuclear expression in CRC cells but negative expression in normal colorectal mucosa of the same sample (original magnification, $\mathrm{x} 100$ and $\mathrm{x} 400$ ); ( $\mathrm{g}$ and $\mathrm{h}$ ) positive expression in CRC metastasis but negative expression in normal lymph nodes of the same sample (original magnification, $\mathrm{x} 100$ and $\mathrm{x} 400$ ).
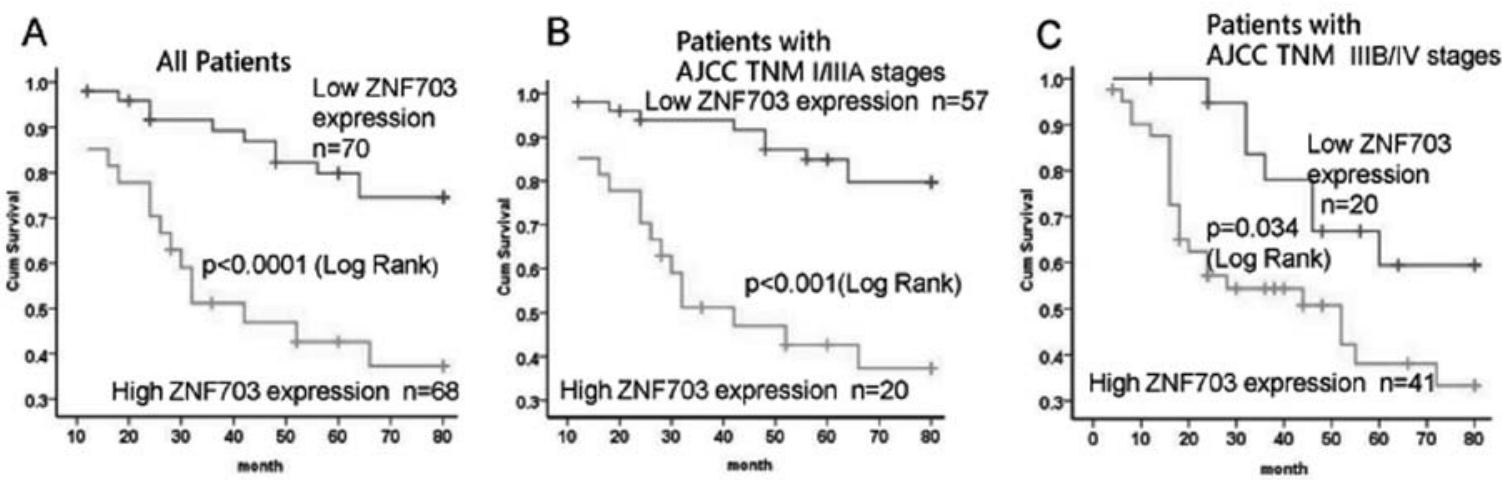

Figure 3. Kaplan-Meier survival analysis of CRC patients in relation to ZNF703 protein expression. The log-rank test was used to calculate P-values: (A) survival of all patients ( $\mathrm{P}<0.001, \log$ Rank); (B) patients in stage AJCC I-IIIA of CRC ( $\mathrm{P}<0.001$, Log Rank); and (C) patients in stage AJCC IIIB-IV of CRC (P=0.034, Log Rank).

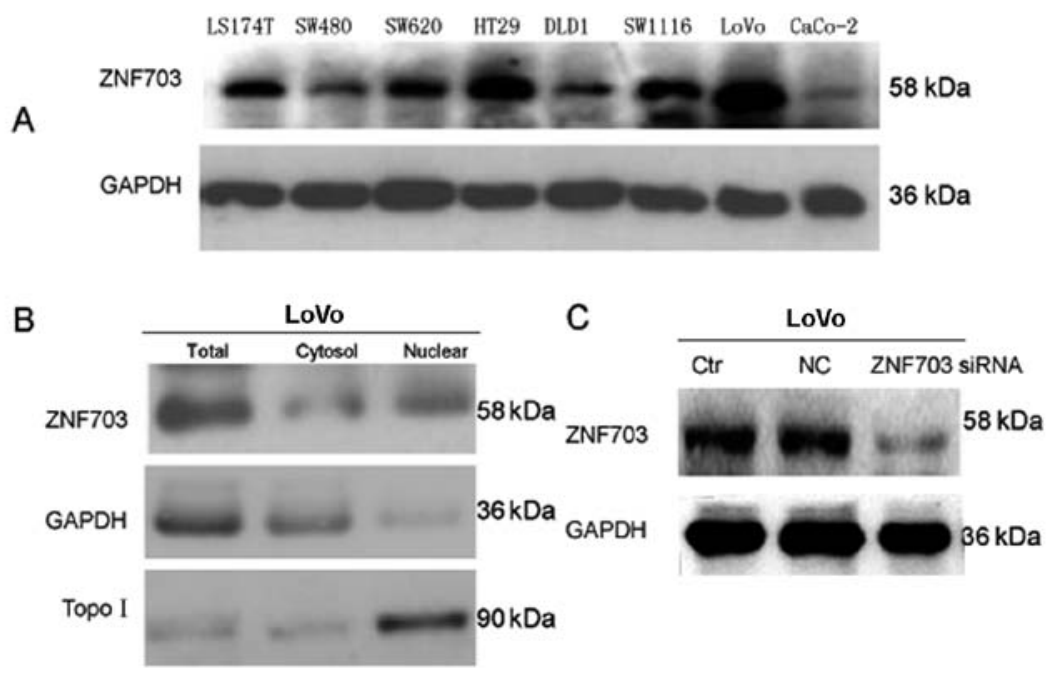

Figure 4. ZNF703 protein expression in cell lines analyzed by western blot analysis. (A) ZNF703 expression in the CRC cell lines (LS174T, SW480, HT29, SW620, DLD1, SW1116, LoVo and CaCo-2). (B) ZNF703 was expressed in the cytoplasm, and nucleus of LoVo cells. (C) ZNF703 expression was inhibited in LoVo cells by transfection with si-ZNF703 siRNA but not by transfection with the negative control siRNA. 
Table I. ZNF703 expression and clinicopathological parameters of the 138 CRC patients.

\begin{tabular}{|c|c|c|c|c|c|}
\hline \multirow[b]{2}{*}{ Parameters } & \multicolumn{3}{|c|}{ ZNF703 expression } & \multirow[b]{2}{*}{$\chi^{2}$} & \multirow[b]{2}{*}{ P-value } \\
\hline & $\begin{array}{c}\text { Total } \\
\mathrm{n}\end{array}$ & $\begin{array}{c}\text { Score } \\
\leq 4 \\
\mathrm{n}(\%)\end{array}$ & $\begin{array}{c}\text { Score } \\
>6 \\
\mathrm{n}(\%)\end{array}$ & & \\
\hline Age (years) & & 70 & 68 & 2.585 & 50.108 \\
\hline$\leq 60$ & 84 & $38(45.2)$ & $46(54.8)$ & & \\
\hline$>60$ & 54 & $32(59.3)$ & $22(40.7)$ & & \\
\hline
\end{tabular}

Gender

Male

$88 \quad 48(54.5) \quad 40(45.5) \quad 1.4190 .234$

Female

$5022(44.0) 28(56.0)$

Tumor location

Colonic and

ileocecal

Rectal

$77 \quad 41(53.2) \quad 36(46.8) \quad 0.443 \quad 0.5006$

$\begin{array}{lll}61 & 29(47.5) & 32(52.5)\end{array}$

Tumor size $(\mathrm{cm})$
$>3$
$7531(41.3) 44(58.7)$
5.7970 .016
$\leq 3$
$6339(61.9) 24(38.1)$

Pathological grading

Well

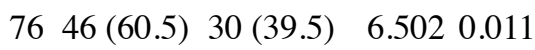

Serosal invasion
Present
$80 \quad 34(42.5) \quad 46(57.7)$
5.1510 .023
Absent
$5836(62.1) 22(37.9)$

Lymph node

metastasis

Present

$\begin{array}{lll}62 & 23(37.1) & 39(62.9)\end{array}$

Absent

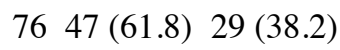

8.3650 .004

AJCC TNM stages

I-IIIA

$78 \quad 50$ (64.1) $27(35.9) \quad 14.0740 .000$

IIIB-IV

$6020(33.3) 41(66.7)$

Tissues

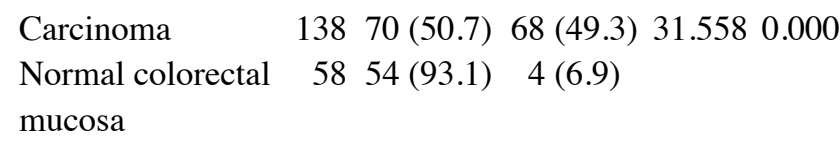

associated with tumor size $(\mathrm{P}=0.016)$, pathological grading $(\mathrm{P}=0.011)$, serosal invasion $(\mathrm{P}=0.023)$, lymph node metastasis $(\mathrm{P}=0.004)$ and AJCC stage $(\mathrm{P}<0.001)$. In addition, we examined the association between ZNF703 expression and cancer-specific survival using a Kaplan-Meier curve and log-rank test. The difference in patient survival between ZNF703-negative and weakly positive tumors was not significant. Patients in these two categories were then combined and resubjected to Kaplan-Meier analysis in comparison with patients with high ZNF703 expression. Patients with high ZNF703 expression had significantly shorter cancer-specific survival than patients with either negative or low ZNF703 expression $(\mathrm{P}<0.001$ for

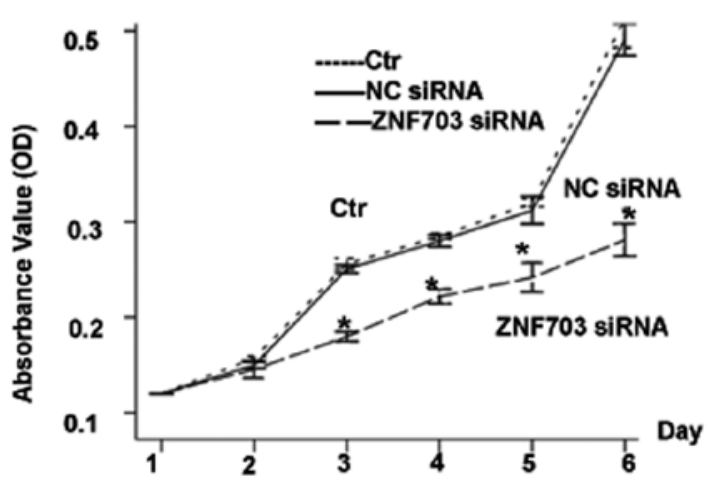

Figure 5. In vitro silencing of ZNF703 inhibits cell proliferation. Data are presented as mean $\pm \mathrm{SD}\left(\mathrm{n}=3,{ }^{*} \mathrm{P}<0.01\right.$ by t-test, comparison of ZNF703 siRNA-transfected cells and negative control siRNA-transfected cells) of the absorbance optical density (OD) of LoVo cells. Significant differences in the OD values among days $3,4,5$ and 6 were not ed.

all patients in Fig. 3A). This relationship was more obvious in patients with AJCC stage I-IIIA CRC $(\mathrm{P}<0.001$; Fig. 3B) than in AJCC stage IIIB-IV patients ( $\mathrm{P}=0.034$; Fig. 3C). Unfortunately, the prognostic value of ZNF703 expression was not evident in patient subgroups stratified according to AJCC stage IIIB-IV (Fig. 3). Furthermore, multivariate analysis failed to confirm overexpression of ZNF703 as an independent prognostic factor for CRC.

ZNF703 protein expression in cell lines. Next, we analyzed ZNF703 expression and function at the cellular level. Expression of ZNF703 in CRC cell lines (LS174T, SW480, HT29, SW620, DLD1, SW1116, LoVo and CaCo-2) was examined by western blot analysis (Fig. 4A). The highest ZNF703 expression was detected in LoVo cells (Fig. 4A). Therefore, LoVo cells were selected for futher experiments.

Based on the results of IHC staining in tumor cells, we decided to examine expression of ZNF703 in protein extracts of LoVo cell, cytoplasm and nuclei by western blot analysis (Fig. 4B). As expected, ZNF703 was expressed in LoVo cell, cytoplasm and nuclei.

In vitro silencing of ZNF703 affects the function of LoVo cells. We investigated the role of ZNF703 in the display of aggressive phenotypes of CRC cells in vitro. siRNA transfection was employed to knock down ZNF703 expression in LoVo cells with high endogenous ZNF703 expression, and high proliferation and invasion capability. Western blot analysis showed $80 \%$ knockdown of the ZNF703 protein when compared to cells treated with negative control siRNA or to untreated cells (Fig. 4C).

As shown by MTT assays, ZNF703 knockdown inhibited cell proliferation when compared with the control cells $(\mathrm{P}<0.0001)$ (Fig. 5). We also used a wound-healing assay to examine the impact of ZNF703 expression on migration of LoVo cells. ZNF703 inhibited the migration of LoVo cells that had been physically wounded and incubated for 24 or $48 \mathrm{~h}$ (Fig. 6A). To analyze invasiveness, another important feature of malignant cells, we performed Transwell invasion assays using cell culture inserts covered with extracellular matrix components; ZNF703/RNAi-transfected cells had relatively weak invasive abilities (Fig. 6C). Collectively, the wound-healing 
A
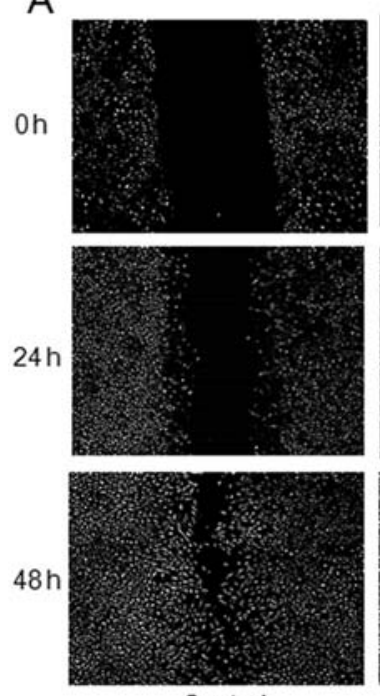

Control

C

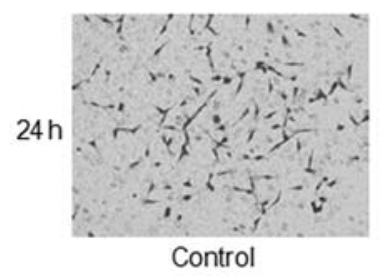

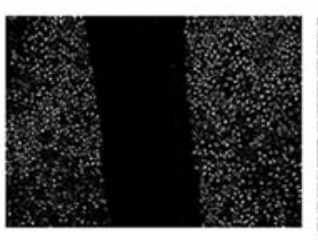
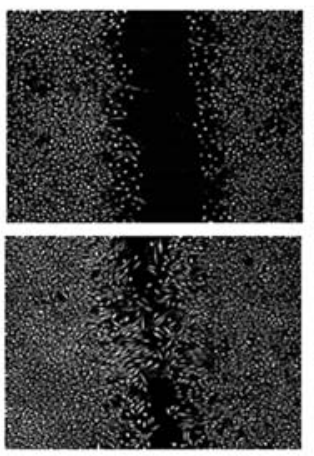

NC siRNA

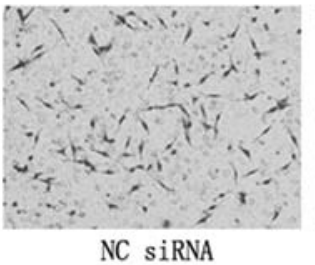

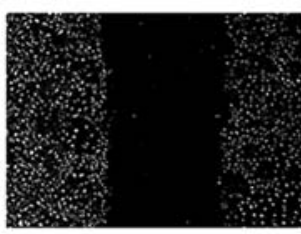
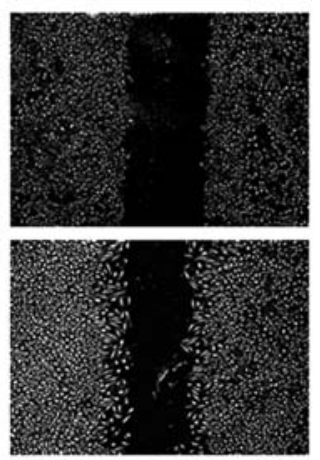

ZNF703 siRNA

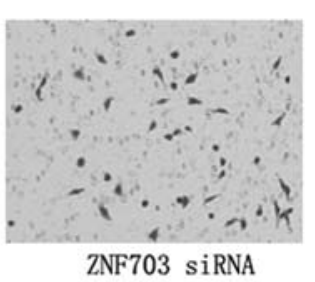

B
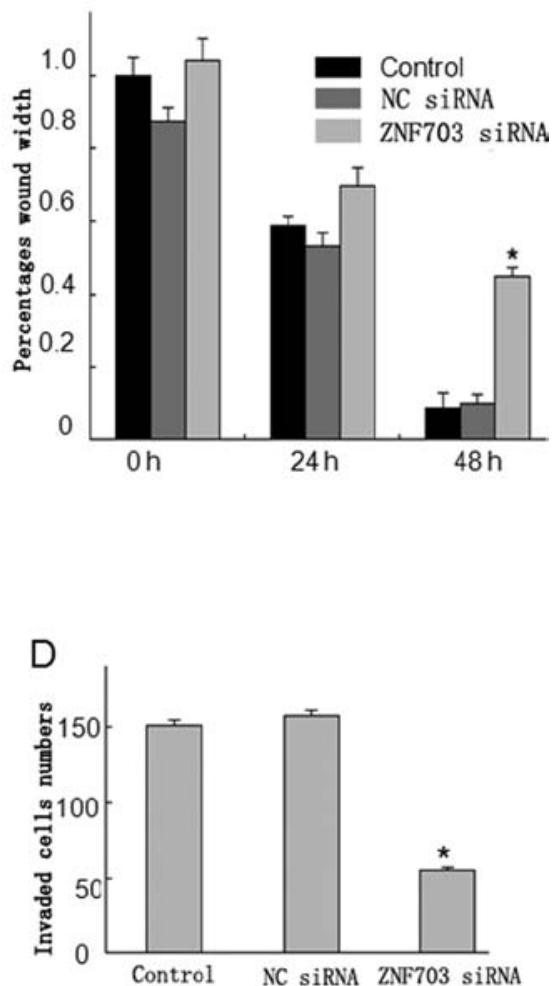

Figure 6. Inhibition of ZNF703 expression facilitates the migration and invasion of LoVo cells. Migration and invasion of cells treated with ZNF703 siRNA (or negative control siRNA) were analyzed at $48 \mathrm{~h}$ post-infection. (A) Wound-healing assays of cells treated with blank control, negative control siRNA or ZNF703 siRNA. Migration of cells to the wound was visualized at 24 and $48 \mathrm{~h}$ with an inverted Leica phase-contrast microscope (original magnification, $\mathrm{x} 100$ ). (B) A histogram reveals the relative migratory distances of the cells. Data are presented as means $\pm \mathrm{SEM}$ ( $\mathrm{n}=3$, ${ }^{*} \mathrm{P}<0.01$ by $\mathrm{t}$-test, comparison of the blank control and negative control siRNA-transfected cells). (C) Transwell assays of cells treated with blank control, negative control siRNA or ZNF703 siRNA. The ZNF703 siRNA-transfected cells showed lower invasion rates compared with blank controls and negative control siRNA-transfected cells. (D) The number of cells that invaded through the membrane was counted in eight fields under a x10 objective lens. Data are presented as mean \pm SEM from 3 independent experiments. Bars, SD. ${ }^{*} \mathrm{P}<0.01$ by t-test.

and invasion assays indicated that downregulation of ZNF703 expression inhibited migration and invasion of LoVo cells.

\section{Discussion}

ZNF703 is an oncogenic transcription factor that regulates expression of numerous genes involved in multiple aspects of the cancer phenotype, including proliferation, increased selfrenewal and invasion (13-17).

The aim of the present study was to examine the role of ZNF703 in CRC. According to our results, ZNF703 expression was higher in CRC tissues than in normal colorectal mucosa at both the mRNA and protein levels. IHC staining revealed that the subcellular localization of ZNF703 was mainly in the nucleus, and partly in the cytoplasm or membrane of CRC cells. In addition, elevated ZNF703 expression was correlated with serosal invasion, lymph node metastasis and AJCC stage. CRC patients with low ZNF703 expression had higher survival rates. At the cellular level, CRC cell lines had higher ZNF703 expression than the normal $297 \mathrm{~T}$ cell line. Due to their relatively high ZNF703 expression, LoVo cells were chosen for siRNA silencing of ZNF703. Based on these knockdown experiments, ZNF703 silencing led to reduced cell prolifera- tion and migration. Collectively, these findings indicated that ZNF703 may not be important in the differentiation of cancer cells, but may play an important role in the progression and metastasis of CRC.

Our findings in regards to the role of ZNF703 in CRC are in agreement with the results of a previous study examining the role of ZNF703 in breast cancer. According to the study of Zhang et al (17), ZNF703 is overexpressed in a number of breast cancer cell lines, while its expression is low in normal mammary epithelial cells. In addition, high ZNF703 expression contributes to tumor aggressiveness (17). The mechanisms leading to ZNF703 overexpression in human tumors are not well established, and it still remains to be determined how ZNF703 expression or function is regulated. In addition, major downstream effectors of oncogenic functions of ZNF703 still remain elusive.

ZNF703 plays a role in tamoxifen resistance induced by activation of the Akt/mTOR signaling pathway and downregulation of $\mathrm{ER} \alpha$, providing a potential mechanism of its action in tumorigenesis (18). In addition, ZNF703 regulates transcription in mouse EpH4.9 cells, complexing with Groucho and repressing E-cadherin, Wnt and TGF- $\beta$ reporter expression (19). Possible mechanisms of its activation include 
point mutation, gene amplification, gene rearrangement and insertion of strong promoter or enhancer. Epigenetic modifications including demethylation and deacetylation may also be responsible for activating ZNF703 in tumorigenesis. Nevertheless, the exact mechanisms leading to ZNF703 oncogenic activation have yet to be examined.

In conclusion, ZNF703 was upregulated in CRC patients, particularly in those with metastatic disease, implying an involvement in poor clinical outcomes. Although the molecular mechanism of ZNF703 action in carcinogenesis remains unexplored, we found that silencing ZNF703 inhibited cancer cell growth and migration in vitro. Hence, ZNF703 should be considered as a potential therapeutic target for metastatic colorectal disease.

\section{Acknowledgements}

The present study was supported by grants from the Guangdong Provincial Science and Technology Projects (nos. 2010B031600243, 2011B05040009 and 2012B050600020) and the National Natural Science Foundation of China (no. 81272761).

\section{References}

1. Ferlay J, Shin HR, Bray F, Forman D, Mathers C and Parkin DM: Estimates of worldwide burden of cancer in 2008: GLOBOCAN 2008. Int J Cancer 27: 2893-2917, 2010.

2. Jemal A, Siegel R, Xu J and Ward E: Cancer statistics, 2010. CA Cancer J Clin 60: 277-300, 2010.

3. Tänzer M, Liebl M and Quante M: Molecular biomarkers in esophageal, gastric, and colorectal adenocarcinoma. Pharmacol Ther 140: 133-147, 2013.

4. Vogelstein B, Fearon ER, Hamilton SR, et al: Genetic alterations during colorectal-tumor development. N Engl J Med 319: 525-532, 1988

5. Santarius T, Shipley J, Brewer D, Stratton MR and Cooper CS: A census of amplified and overexpressed human cancer genes. Nat Rev Cancer 10: 59-64, 2010.

6. Pereira-Castro I, Costa AM, Oliveira MJ, Barbosa I, Rocha AS, Azevedo L and da Costa LT: Characterization of human NLZ1/ ZNF703 identifies conserved domains essential for proper subcellular localization and transcriptional repression. J Cell Biochem 114: 120-133, 2013.
7. Garcia MJ, Pole JC, Chin SF, et al: A1 Mb minimal amplicon at 8p11-12 in breast cancer identifies new candidate oncogenes. Oncogene 24: 5235-5245, 2005.

8. Gelsi-Boyer V, Orsetti B, Cervera N, et al: Comprehensive profiling of 8p11-12 amplification in breast cancer. Mol Cancer Res 3: 655-667, 2005.

9. Chin K, DeVries S, Fridlyand J, et al: Genomic and transcriptional aberrations linked to breast cancer pathophysiologies. Cancer Cell 10: 529-541, 2006.

10. Adélaïde J, Finetti P, Bekhouche I, et al: Integrated profiling of basal and luminal breast cancers. Cancer Res 67: 11565-11575, 2007.

11. Kwek SS, Roy R, Zhou H, Climent J, Martinez-Climent JA, Fridlyand $\mathbf{J}$ and lbertson DG: Co-amplified genes at 8p12 and $11 \mathrm{q} 13$ in breast tumors cooperate with two major pathways in oncogenesis. Oncogene 28: 1892-1903, 2009.

12. Melchor L, Garcia MJ, Honrado E, et al: Genomic analysis of the 8p11-12 amplicon in familial breast cancer. Int J Cancer 120: 714-717, 2007.

13. Haverty PM, Fridlyand J, Li L, et al: High-resolution genomic and expression analyses of copy number alterations in breast tumors. Genes Chromosomes Cancer 47: 530-542, 2008.

14. Sircoulomb F, Nicolas N, Ferrari A, et al: ZNF703 gene amplification at $8 \mathrm{p} 12$ specifies luminal B breast cancer. EMBO Mol Med 3: 153-166, 2011.

15. Holland DG, Burleigh A, Git A, et al: ZNF703 is a common Luminal B breast cancer oncogene that differentially regulates luminal and basal progenitors in human mammary epithelium. EMBO Mol Med 3: 167-180, 2011.

16. Curtis C, Shah SP, Chin SF, Turashvili G, Rueda OM and Dunning MJ: The genomic and transcriptomic architecture of 2,000 breast tumours reveals novel subgroups. Nature 486: 346-352, 2012.

17. Zhang X, Mu X, Huang O, Xie Z, Jiang M, Geng M and Shen K: Luminal breast cancer cell lines overexpressing ZNF703 are resistant to tamoxifen through activation of Akt/mTOR signaling. PLoS One 8: e72053, 2013.

18. Reynisdottir I, Arason A, Einarsdottir BO, et al: High expression of ZNF703 independent of amplification indicates worse prognosis in patients with luminal B breast cancer. Cancer Med 2: 437-446, 2013.

19. Slorach EM, Chou J and Werb Z: Zeppol is a novel metastasis promoter that represses E-cadherin expression and regulates p120-catenin isoform expression and localization. Genes Dev 25: 471-484, 2011. 